\title{
The presence of joint predictors generates conjunctive predictions
}

\author{
Ru Qi Yu ${ }^{1}$ - Jiaying Zhao ${ }^{1,2}$
}

Published online: 23 July 2020

(C) The Psychonomic Society, Inc. 2020

\begin{abstract}
The cognitive system readily detects statistical relationships where a cue predicts a specific outcome. What is less known is how the mind generates predictions when multiple cues are presented simultaneously that predict different outcomes. Here, we examine the nature of such predictions. Specifically, we examine whether the presence of joint cues leads to conjunctive predictions that represent the overlap between the outcomes associated with the cues, or disjunctive predictions that represent the total possible outcomes. To test this, we used a visual search paradigm where participants first viewed a cue and then searched for a target in an array. Each cue predicted where the target would appear in one of the four quadrants in the array. After learning the cue-location associations, two cues were presented simultaneously, and participants searched for the target where the target now appeared in each quadrant with equal probability. We found that participants were faster to find the target in conjunctive locations over disjunctive ones upon seeing two cues (Experiment 1). This attentional prioritization was not solely driven by the smaller spatial scope of conjunctive locations (Experiment 2), and was present when two cues were presented in two feature dimensions in a single object (Experiment 3). These results were consistent with a conjunctive account, where the presence of joint cues led to a conjunctive prediction that represented the overlap of the different outcomes associated with each cue. The study sheds a new light on how the mind makes predictions in novel contexts based on existing knowledge.
\end{abstract}

Keywords Statistical learning $\cdot$ Predictions $\cdot$ Reasoning $\cdot$ Visual search

The ability to accurately predict an outcome based on a preceding cue is important for not only meeting survival needs (e.g., knowing what color of food is safe to eat) but also preparing for future circumstances (e.g., seeking shelter when seeing dark clouds). The mind readily acquires these cueoutcome associations via a number of learning mechanisms, such as conditioning (Mackintosh, 1974; Rescorla \& Wagner, 1972), associative learning (Fanselow \& Poulos, 2005; Le Pelley, 2004), and statistical learning (Fiser \& Aslin, 2001; Saffran, Aslin, \& Newport, 1996).

What is less known is how the mind makes predictions in a new context where two cues are jointly presented that were previously associated with different outcomes. For example, smoking is associated with cardiovascular and lung diseases,

Jiaying Zhao

jiayingz@psych.ubc.ca

1 Department of Psychology, Institute for Resources, Environment and Sustainability, University of British Columbia, Vancouver, BC V6T 1Z4, Canada

2 Institute for Resources, Environment and Sustainability, University of British Columbia, Vancouver, BC, Canada while alcohol consumption is associated with cardiovascular and brain diseases. What do people infer when both smoking and drinking are present? A conjunctive prediction raises an expectation that represents the common property of the outcomes associated with both cues (i.e., cardiovascular problems), whereas a disjunctive prediction generates expectations that contain all possible outcomes associated with both cues (i.e., cardiovascular problems, lung diseases, and brain diseases). While both predictions are logically plausible, it is currently unknown which prediction is generated in the mind. Answers to this question can shed light on how the cognitive system makes predictions in novel contexts based on existing knowledge. It can also help elucidate reasoning biases such as the conjunction fallacy, where people mistakenly judge a conjunctive statement to be more probable than a disjunctive one (Tversky \& Kahneman, 1983).

In the current study, we examined how predictions were made when multiple cues associated with different outcomes were presented together. Specifically, we examined whether the presence of joint cues would lead to conjunctive predictions that represented the overlap between the different outcomes associated with the cues, or disjunctive predictions that represented the total possible outcomes associated with the 
cues. In past studies on implicit learning and visual search, target search has been found to be faster if there were reliable but incidental cues predicting the target location. For example, target search was faster when the configuration of the search array predicted target location (Chun \& Jiang, 1998; Jungé, Scholl, \& Chun, 2007). In the study, we used a similar visual search paradigm. Specifically, participants first viewed a cue (e.g., a blue dot) and then searched for a target (e.g., a rotated $T)$ in an array. Each cue predicted where the target would appear in one of the four quadrants in the array (e.g., the blue circle meant that the target would always appear in the top half of the array, and the red circle meant that the target would always appear in the left half of the array). After being exposed to the cue-location associations, participants completed a prediction phase where two cues were now jointly presented, and participants searched for the target, which could appear in each quadrant in the array with equal probability. In other words, the two cues were no longer predictive of the target location, but the attentional prioritization of a conjunctive or disjunctive region in the array would indicate an expectation of the target to appear there, thus indicating the nature of predictions made by the participants.

\section{Experiment 1}

Experiment 1 examined what type of predictions participants would make when they saw a pair of cues, after learning that each cue was associated with a different outcome.

\section{Method}

\section{Participants}

To determine the sample size, we conducted a power analysis using G*Power (Faul, Erdfelder, Lang, \& Buchner, 2007) based on the effect size of $n_{p}{ }^{2}$ of 0.19 from a similar paradigm on statistical learning that used response time as the measure (Zhao \& Luo, 2017). Given this effect size and the mixed design in the current experiment, a minimum of 104 participants were required to achieve $95 \%$ power. Thus, a total of 120 students ( 81 females, $M_{\text {age }}=20.0$ years, $S D=2.3$ ) from the University of British Columbia (UBC) participated in this experiment for course credit. All participants reported normal or corrected-to-normal vision.

\section{Stimuli}

In Experiment 1, the cues were four colored circles (RGB): blue $(0,0,255)$, red $(255,0,0)$, yellow $(255,255,0)$, and gray $(192,192,192)$, each subtending $2.2^{\circ}$ of visual angle. Each circle was followed by a search array that contained 16 objects appearing in four quadrants (see Fig. 1a). Each quadrant was an invisible $4 \times 4$ grid, with each cell subtending $1.7^{\circ}$ of visual angle. There was a gap subtending $2.2^{\circ}$ of visual angle between any two adjacent quadrants. Four objects appeared in each quadrant, where no row or column in the grid could be empty. One object was the target (a rotated $T$ ), and the other 15 objects were $L$-shaped distractors.

\section{Procedure}

There were two phases in the experiment. Participants first completed the exposure phase, where in each trial one colored circle appeared on the screen for 1,000 ms, followed by a $1,000 \mathrm{~ms}$ blank screen, and then a search array. The task was to find the target and indicate which direction it was pointing (left or right) by pressing a key as quickly and accurately as possible. Unbeknownst to the participants, the color of the circle predicted in which half of the array the target would appear. For example, after the blue circle, the target would always appear in the top half of the array (Fig. 1a). The target appeared in a random cell within the quadrants, and the direction of the target was also random. The four color-location associations were determined randomly for each participant, but remained fixed throughout exposure. Each color circle and its associated search array were presented 40 times during exposure, resulting in 160 trials, presented in a random order. The search array appeared on the screen until response. The intertrial interval was $1,000 \mathrm{~ms}$.

After exposure, participants completed the prediction phase, which was identical to exposure, except that now, two colored circles were presented simultaneously, followed by a search array, where the target now appeared in each of the four quadrants with equal likelihood (Fig. 1b). The four colored circles created six unique color pairs. Each color pair was presented four times, resulting in 24 trials presented in a random order. Because there were four trials of each unique color pair, the target appeared in every quadrant following the color pair. In other words, the colored circles were no longer relevant to the search task. The limited number of trials during the prediction phase was to minimize the degradation of learning during the exposure phase, since the colors were no longer predictive of the target locations. The two circles in each pair were presented either horizontally or vertically (randomly determined), with the order of the two colors counterbalanced.

Based on the color-location associations during exposure, there were three location types following each pair (Fig. 1b): locations consistent with a conjunctive prediction, locations consistent with an exclusive disjunctive prediction, and impossible locations where the target never appeared based on exposure. For example, following the blue-red pair, the top left quadrant was a conjunctive location, the top right and bottom left quadrants were disjunctive locations, and the bottom right quadrant was an impossible location. Because the target now appeared in each of the four 
a) Exposure phase

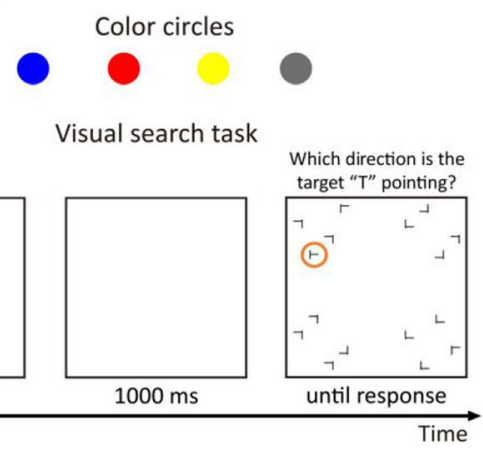

Color-location associations
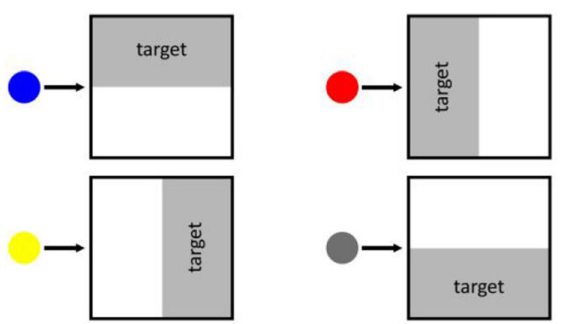

c) Target search performance

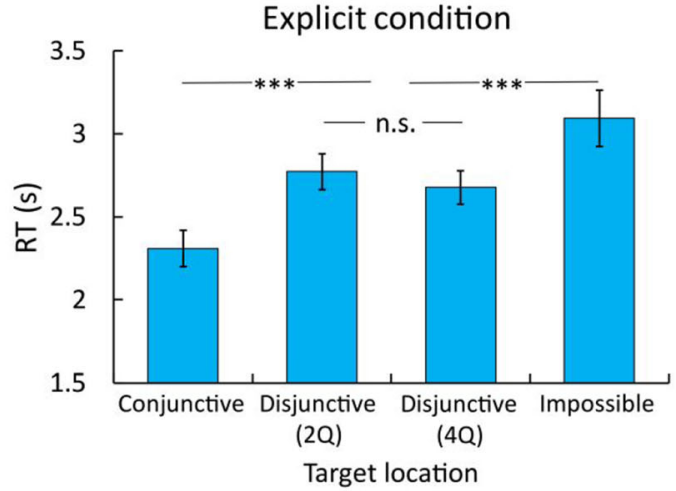

Fig. 1 Experiment 1. a During exposure, participants saw a color circle first, then searched for the target (the rotated $T$ ) and judged its direction as quickly and accurately as possible. Each color circle predicted in which half of the search array the target would appear (indicated in gray). b During prediction, there were six color pairs. Each pair was presented first, followed by a search array. The target now appeared in all four quadrants with equal probability. There were three location types: locations consistent with a conjunctive prediction (C), locations

quadrants with equal probability, faster response time in a given location would reveal the nature of predictions formed by participants upon seeing the color pair. For example, faster response time in the conjunctive than in the disjunctive location suggests that participants expected the target to appear in the conjunctive location, thus indicating a conjunctive prediction. However, if response time was identical in the conjunctive and disjunctive locations, this suggests no expectation of the target appearing in the conjunctive location, which would indicate a lack of the conjunctive prediction. b) Prediction phase

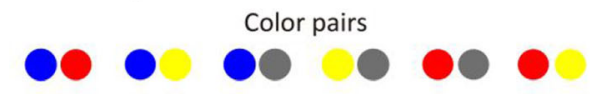

Visual search task

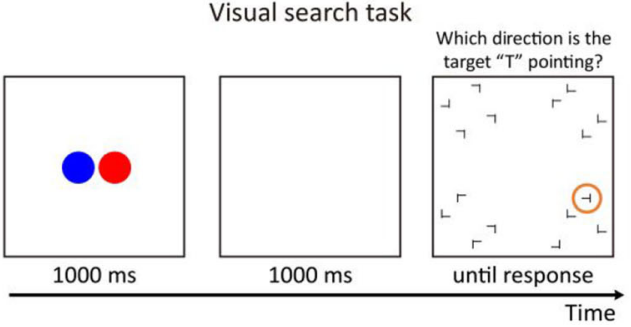

Location types during prediction
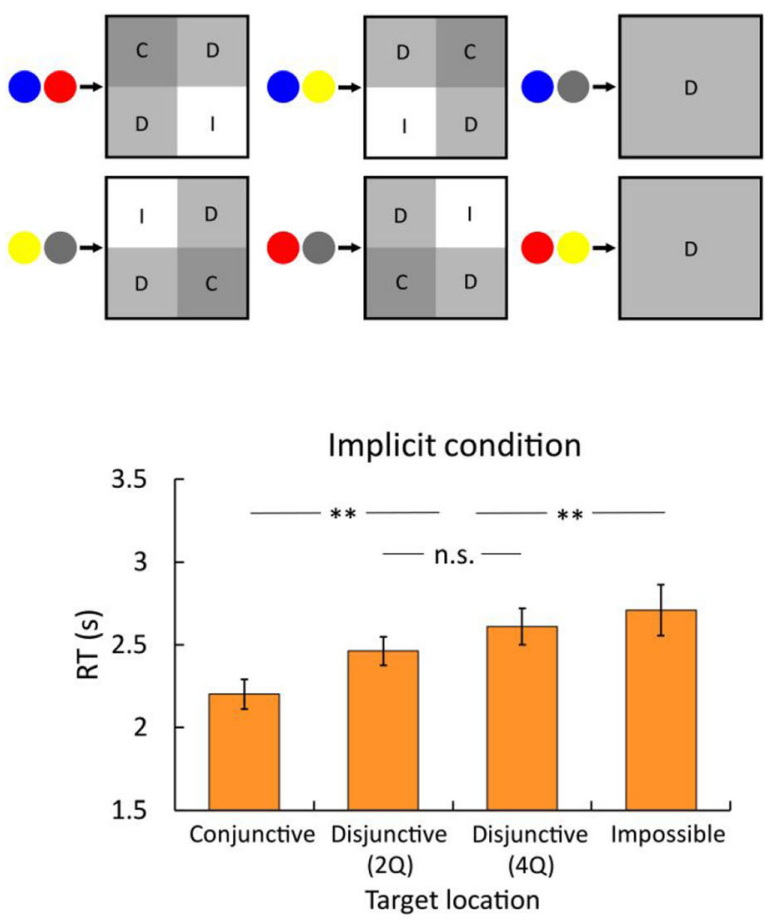

consistent with an exclusive disjunctive prediction (D), and impossible locations (I). For four color pairs, there were two disjunctive quadrants (2Q); for the other two pairs, there were four disjunctive quadrants (4Q). c Response time (RT) in seconds (s) of visual search for each location type was graphed for the explicit condition where participants were told about the color-location associations, and the implicit condition where participants were not told about the associations (error bars reflect $\pm 1 S E M$ ). ${ }^{* *} p$ $<.01$. $* * * p<.001$. (Color figure online)

To examine whether such predictions required explicit knowledge of the color-location associations, half of the participants $(N=60)$ were randomly assigned to the explicit condition, where they were told about the color-location associations (i.e., where the target would appear following which color), and the other half were in the implicit condition, where they were told to only pay attention to the colored circles, and there was no mention of the associations. During the prediction phase, participants in both conditions were told that now they would see two colors, but their task was to find the target in the array as quickly and accurately as possible. 
After the prediction phase, participants in the implicit condition also completed a test phase to probe their awareness of the color-location associations. Specifically, they completed four trials where they were asked to choose in which half (top, bottom, left, or right) of the array the target would appear after seeing each color circle.

\section{Results and discussion}

Response time (RT) of correct trials in the prediction phase was analyzed to examine what kind of predictions participants made when they saw the color pair (Fig. 1c). Search accuracy was high $(>97 \%)$ in all four types of locations and there was no reliable difference in accuracy across conditions and locations, suggesting no speed-accuracy trade-off (see Appendix 1 for the detailed analysis on the search accuracy across experiments).

A 2 (condition: explicit vs. implicit; between subjects) $\times 4$ (location type: conjunctive, two-quadrant disjunctive, four-quadrant disjunctive, and impossible; within subjects) mixed-design analysis of variance (ANOVA) revealed a significant main effect of location type, $F(3,354)=16.04, p<.001, \eta_{\mathrm{p}}^{2}=0.12$, but no main effect of condition, $F(1,118)=3.27, p=.07, \eta_{\mathrm{p}}{ }^{2}$ $=0.03$, or interaction, $F(3,354)=1.29, p=.28, \eta_{\mathrm{p}}{ }^{2}=$ 0.01 . Based on the post hoc Tukey HSD test, there was no significant difference between the two-quadrant and four-quadrant disjunctive trials ( $p \mathrm{~s}>.63)$, so they were combined into a single group of disjunctive trials, and we performed planned contrasts separately for the explicit and implicit conditions. For both conditions, RT was significantly faster when the target appeared in conjunctive locations than in disjunctive locations $(p s<$ $.01)$. RT was also faster when the target appeared in disjunctive locations than in impossible locations ( $p$ s $<$ $.01)$. This suggests that participants expected the target to appear in conjunctive locations more strongly than in disjunctive locations, thus indicating a conjunctive prediction, regardless of whether the knowledge about the color-location associations were explicitly told or implicitly learned. Moreover, in the implicit condition, test accuracy was $51 \%$, which was reliably above chance ( $p$ $<.001$ ), indicating successful learning of the colorlocation associations. These results suggest that when two cues were presented simultaneously, participants made a conjunctive prediction of the outcome, which was the overlap of the different outcomes associated with each cue.

Another way to gauge learning in the exposure phase in the implicit condition was to compare the search RT in the implicit condition with that in the explicit condition. If participants in the implicit condition improved more over the course of exposure in search RT than those in the explicit condition, then this would provide some evidence of implicit learning. However, this timecourse analysis could be confounded by a possible ceiling effect (i.e., participants reaching a fast search RT early on) in the explicit condition. Nevertheless, the search RT data in the exposure phase were presented in Appendix 2 for all experiments.

\section{Experiment 2}

In Experiment 1, the spatial scope of conjunctive location (one quadrant) was smaller than that of disjunctive location (two or four quadrants), so the faster RT might be explained by the smaller area for search. This account is unlikely for two reasons. First, the conjunctive quadrant was identical in scope to the impossible quadrant, and yet RT was reliably faster in the conjunctive quadrant. Second, there was no difference in RT between the twoquadrant and the four-quadrant disjunctive locations, suggesting that the spatial scope had little impact on the RT. Nonetheless, we aimed to equate the spatial scope of conjunctive and disjunctive locations in Experiment 2.

\section{Method}

\section{Participants}

A new group of 120 students ( 95 females, $M_{\text {age }}=20.2$ years, $S D=1.9)$ from UBC participated for course credit.

\section{Stimuli and procedure}

The stimuli and procedure were identical to those in Experiment 1, except for one important difference: During exposure, each color circle predicted that the target could appear in three of the four quadrants (Fig. 2a). This means that during the prediction phase, two quadrants would be consistent with a conjunctive prediction, and the other two would be consistent with an exclusive disjunctive prediction, following a pair of color circles (Fig. 2b). Thus, the spatial scope of the conjunctive and disjunctive quadrants was equal. During the test phase, participants were again tested for their knowledge of the color-location associations. Specifically, each color was first presented to the participants, and they were given four options. Each option was a unique three-quadrant combination, as in Fig. 2a.

\section{Results and discussion}

As in Experiment 1, we performed a two-way mixeddesign ANOVA on the RTs of correct trials (Fig. 2c). It 
a) Color-location associations
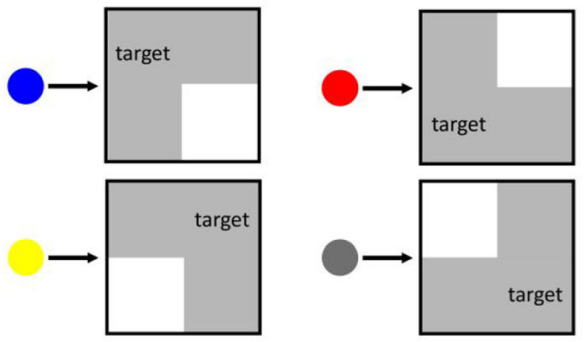

c) Target search performance

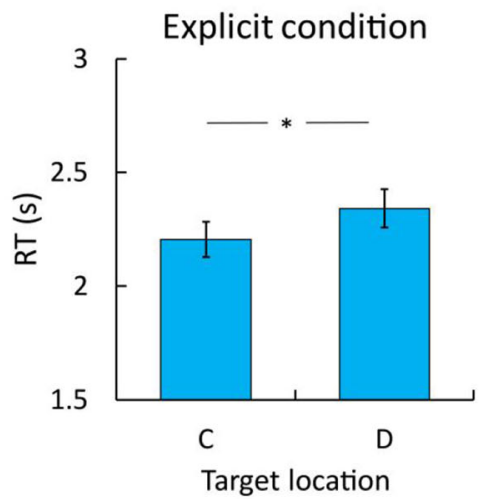

Fig. 2 Experiment 2. a Each color circle now predicted that the target would appear in three quadrants of the search array (indicated in gray) during exposure. b During the prediction phase, two quadrants were consistent with a conjunctive prediction $(\mathrm{C})$, and the other two were

revealed no main effect of condition, $F(1,118)=0.40$, $p=.53, \eta_{\mathrm{p}}{ }^{2}=0.00$, or trial type, $F(1,118)=1.22, p=$ $.27, \eta_{\mathrm{p}}{ }^{2}=0.01$, but a significant interaction between condition and trial type, $F(1,118)=3.865, p=.05$, $\eta_{\mathrm{p}}{ }^{2}=0.03$. In the explicit condition, RT was reliably faster when the target appeared in conjunctive locations than in disjunctive locations, $t(1,59)=2.03, p<.05, d$ $=0.24$, but there was no significant difference in RT between the two locations in the implicit condition, $t(1$, 59) $=0.66, p=.51, d=0.07$. The lack of difference could be explained by a lack of learning of the colorlocation associations in the implicit condition because the test accuracy was $31 \%$, which was not reliably above chance $($ chance $=25 \%, p=.11$ ). These results again suggest a conjunctive prediction, at least when the color-location associations were learned explicitly. This also means that the findings in the experiments were not solely driven by the fact that conjunctive locations were smaller in spatial scope than disjunctive locations.

\section{Experiment 3}

In Experiments 1 and 2, the two colored circles were shown simultaneously during the prediction phase to elicit b) Location types during prediction
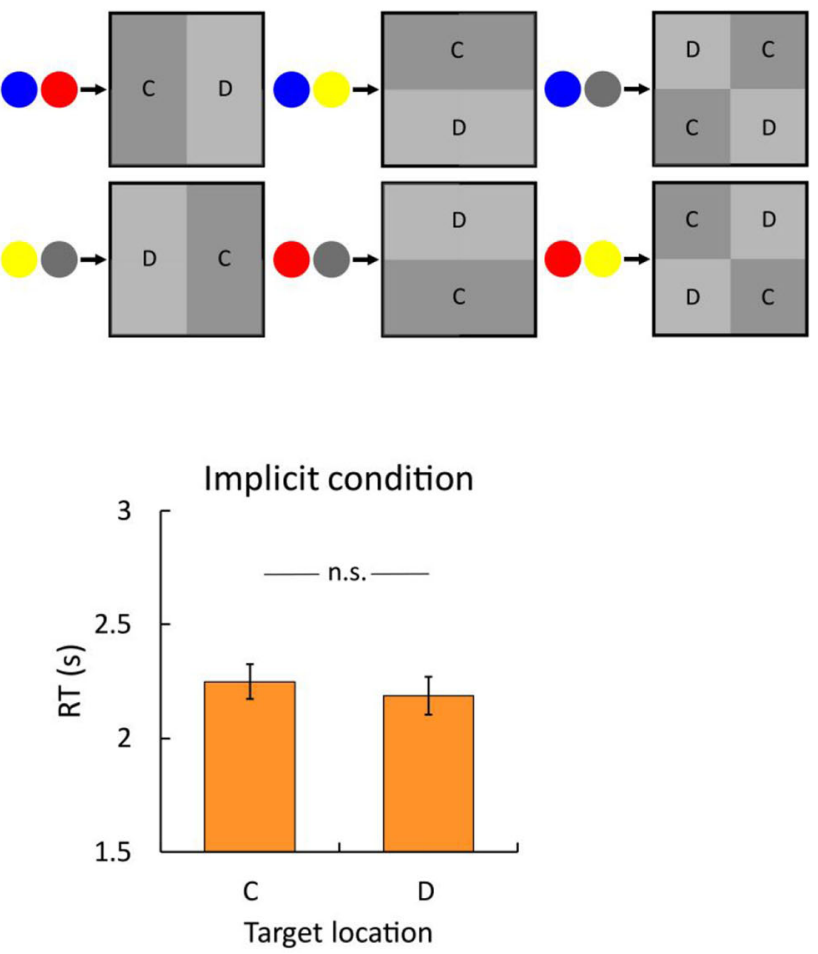

consistent with a disjunctive prediction (D). $\mathbf{c}$ RT of visual search for each location type was graphed for the explicit and implicit conditions (error bars reflect $\pm 1 S E M$ ). ${ }^{*} p<.05$. (Color figure online)

conjunctive predictions. To see if such prediction required the presentation of two separate objects, we tested an alternative method in this experiment. Specifically, we used two independent feature dimensions (color and texture) as cues, which were presented as a single object during the prediction phase (Singer \& Gray, 1995; Treisman \& Gelade, 1980).

\section{Method}

\section{Participants}

A new group of 120 students ( 90 females, $M_{\text {age }}=20.5$ years, $S D=2.8$ ) from UBC participated for course credit.

\section{Stimuli and procedure}

The stimuli and procedure were the same as those in Experiment 1, except for two important differences. First, during exposure there were two color circles (red and blue as in Experiment 1) and two textured circles (dotted and stripy circles; Fig.3a). The textured circles were presented in black (RGB: 0, 0, 0). As before, each circle predicted in which half the target would appear. Second, during prediction, participants saw one circle first, followed by a search array. Each circle contained one of the two colors in one of the two 
textures in the exposure phase (i.e., a blue stripy circle, a blue dotted circle, a red stripy circle, or a red dotted circle; Fig.3b).

\section{Results and discussion}

As in Experiment 1, we performed a two-way mixeddesign ANOVA on the RTs of correct trials (Fig. 3c). It revealed a main effect of condition, $F(1,118)=23.04$, $p<.001, \eta_{\mathrm{p}}^{2}=0.16$, and location type, $F(2,236)=$ $31.06, p<.001, \eta_{\mathrm{p}}{ }^{2}=0.27$, and a reliable interaction, $F(2,236)=22.42, p<.001, \eta_{\mathrm{p}}^{2}=0.12$. In the explicit condition, there was a main effect of location type, $F(2$, 118) $=43.01, \mathrm{p}<.001, \eta_{\mathrm{p}}{ }^{2}=0.42$. Based on the post hoc Tukey HSD test, all pair-wise comparisons were reliable $(p s<.001)$. In the implicit condition, there was still a main effect of location type, $F(2,118)=$ $5.32, p<.001, \eta_{\mathrm{p}}{ }^{2}=0.24$, but the only reliable difference was between conjunctive and impossible locations $(p<.01)$. The other pair-wise comparisons were not reliable $(p s>.11)$. The lack of difference between conjunctive and disjunctive locations could be explained by a lack of learning of the color-location associations in the implicit condition because the test accuracy was $33 \%$, which was not reliably above chance $(p=.07)$.

a) Feature-location associations
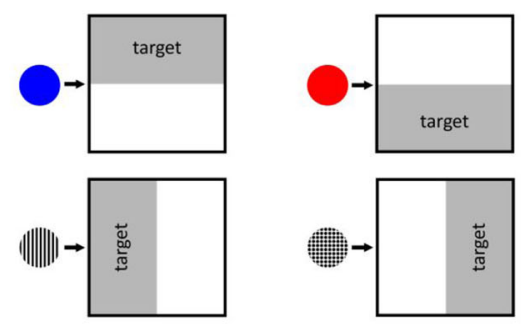

c) Target search performance
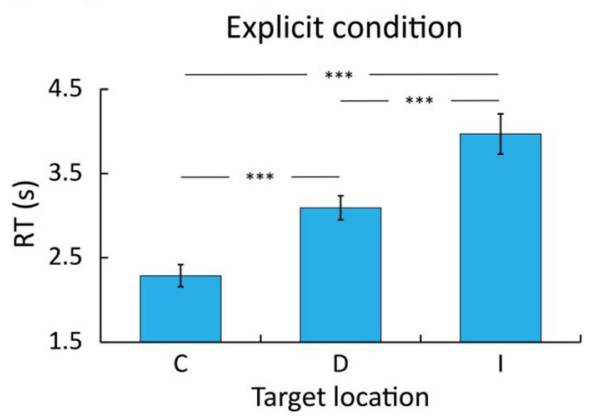

Fig. 3 Experiment 3. a During exposure, each of the two colors and two textures predicted in which half of the search array (indicated in gray) the target would appear. b During the prediction phase, there were four circles made from the combinations of colors and textures. Again, there
These results, again, suggest a conjunctive prediction, at least when the color-location associations were learned explicitly.

\section{General discussion}

The current study examined how people made predictions when seeing a pair of cues after learning that each cue predicted a different outcome. We found that participants prioritized the conjunctive quadrant over disjunctive ones for visual search upon seeing two cues, each of which predicted a different half of the array (Experiment 1). This prioritization was not solely driven by the smaller spatial scope of the conjunctive quadrant (Experiment 2), and was present when two cues were presented in two feature dimensions in a single object (Experiment 3). Prioritizing the conjunctive quadrant implies that participants expected to find the target in the quadrant shared by the two halves that were predicted by the two cues. This suggests that when two cues were presented simultaneously, a conjunctive prediction was made of the outcome that was the overlap of the different outcomes associated with each cue. This

\section{b) Location types during prediction}
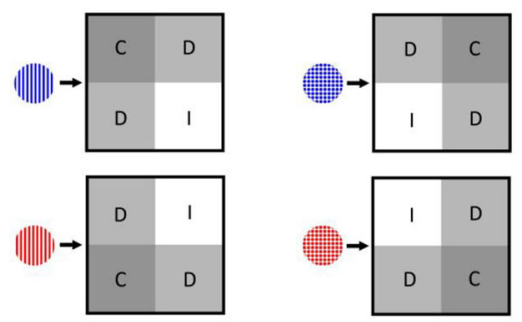

Implicit condition

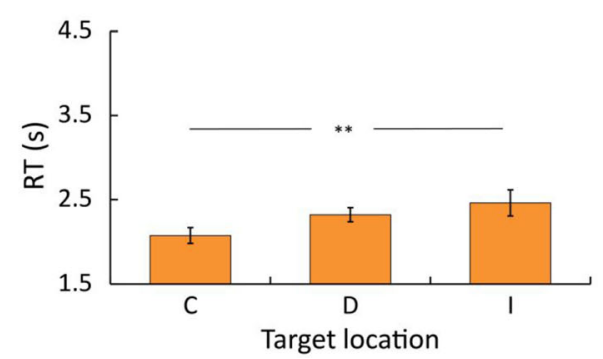

were three location types: the conjunctive (C), disjunctive (D), and impossible (I) locations. c RT of visual search for each location type was graphed for the explicit and implicit conditions (error bars reflect \pm 1 SEM). $* * p<.01 . * * * p<.001$. (Color figure online) 
conjunctive prediction seemed to depend on successful learning of the cue-outcome associations, either through explicit instructions or implicit statistical learning.

This finding was surprising because the simultaneous presentation of the two cues did not necessarily dictate a conjunctive prediction. Participants were not told anything about where the target might appear when they saw the two cues during the prediction phase. In fact, in this phase the target appeared in all quadrants with equal probability. Therefore, the conjunctive prediction seems to spontaneously arise when encountering the two cues. The current finding is different from previous work showing that the joint presence of different sensory stimuli leads to faster response time to the stimulus, an effect known as the intersensory facilitation (e.g., Hershenson, 1962; Nickerson, 1973; Raab, 1962). Instead of facilitating response time generally, here, we show that participants selectively responded to the conjunctive quadrant faster over other quadrants.

The conjunctive prediction can be explained by a summation account where the probability of target appearance in each quadrant is summed up from the previous probability associated with each cue. During exposure, each cue was associated with a probability distribution of target appearance across the search array to guide visual attention (see Fig. 4a), similar to a saliency map (Itti \& Koch, 2001). The simultaneous presentation of the two cues involved summing the two probability distributions associated with the cues (see Fig. 4b),

\section{a) $\operatorname{Pr}(T)$ during exposure}
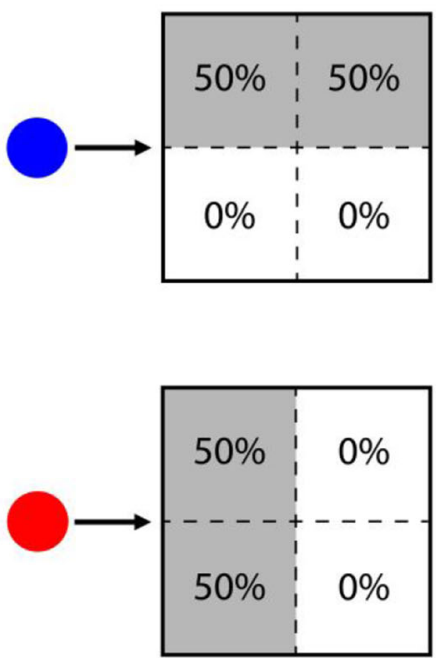

Fig. 4 A summation account of the conjunctive prediction. a Probability distributions of target appearance during exposure in Experiment 1 for blue and red circles as two examples. b Summed and normalized probability distribution from the two previous distributions from blue rendering the conjunctive quadrant as the most likely (50\%) to contain the target, the exclusive disjunctive quadrants as less likely (25\%), and the impossible quadrant as impossible $(0 \%)$. Indeed, using the RT data from Experiment 1, we generated a saliency map (Fig. 4c) that resembled the summed distributions over the four quadrants (Fig. 4b). Moreover, the RT difference between conjunctive and disjunctive quadrants seemed similar to the difference between disjunctive and impossible quadrants in the explicit conditions of Experiments 1 and 3 , closely matching the probability difference between these quadrants. Thus, these data provide initial support for the summation account.

The summation account suggests that people interpreted the simultaneous presentation of the two cues using an additive function where the probability of each cue was added up for each quadrant. The quadrant with the highest probability (the conjunctive quadrant) was then prioritized for subsequent processing (in this case, visual search). We postulate that this summation process can sometimes lead to errors reflected in higher-level reasoning biases such as the conjunction fallacy, where a joint statement containing two cues (e.g., Linda is a bank teller and is active in the feminist movement) is judged as more likely than a statement containing only one cue (e.g., Linda is a bank teller; Mellers, Hertwig, \& Kahneman, 2001; Tversky \& Kahneman, 1983). In this case, the conjunction (Linda is both a bank teller and an active feminist) may draw

\section{b) Summed normalized $\operatorname{Pr}(T)$}

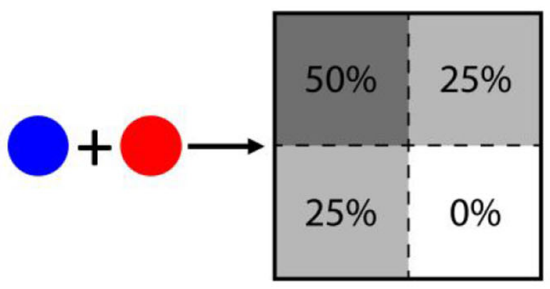

\section{c) RT (s) from Experiment 1}

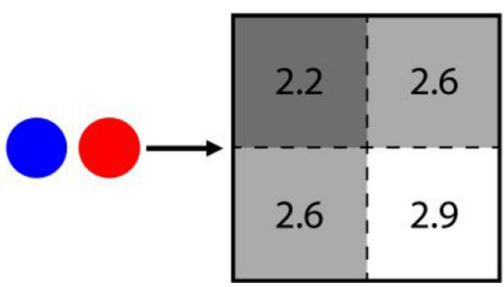

and red circles. Higher probabilities were represented by darker colors. c RT in seconds (s) in each quadrant in the explicit condition in Experiment 1. Faster RTs were represented by darker colors 
more attention than the disjunction (Linda is either a bank teller or an active feminist), therefore causing the estimated conjunctive probability to be higher than the disjunctive probability.

The proposed summation account has not been directly examined in the past literature, but previous work has examined related questions and provided evidence foundational to our summation account. Research in implicit visual learning shows that attention is sensitive to the distribution of probabilities in space (e.g., Jiang, Swallow, Rosenbaum, \& Herzig, 2013). Specifically, spatial attention can be biased toward the location that has the highest probability of containing the search target. Another line of research in sensory integration found that the joint presentation of a visual stimulus and an auditory stimulus led to the unique perceptual experiences that are separate from the experience arising from an individual stimulus, causing multisensory integration effects, such as the McGurk effect (McGurk \& MacDonald, 1976) or the ventriloquist effect (Alais \& Burr, 2004). These studies suggest that visual attention can be drawn to locations with a higher probability of target presence and that multiple cues can generate sensory integration of the different outcomes.

One alternative interpretation of the current findings is that the faster RT in the conjunctive location was driven by a subset of color combinations (e.g., only the blue and red combination facilitated RT). To explore this possibility, we examined the RT for all color combinations in the prediction phase for Experiment 1 (see Appendix 3). The results showed consistent RT profiles across all color combinations, suggesting that the faster $\mathrm{RT}$ in the conjunctive location likely was not driven by a subset of color combinations.

A puzzle in Experiments 2 and 3 was that participants in the implicit condition did not perform above chance in the test of the color-location associations, and yet their search performance was better in the conjunctive location than in the disjunctive locations in Experiment 3, but not in Experiment 2. This discrepancy could be driven by the different levels of difficulty in mentally combining outcome maps. It may be more difficult to combine two different three-quadrant outcome maps in order to distinguish the conjunctive and disjunctive locations in Experiment 2 than to combine two different two-quadrant outcome maps in Experiment 3. This explanation is supported more by the larger RT difference between conjunctive and disjunctive locations in the explicit condition in Experiment 3 than in
Experiment 2. This suggests that despite explicit knowledge of the color-location associations, the search advantage of the conjunctive location was weaker in Experiment 2 when participants had to combine two different three-quadrant maps than it was in Experiment 3.

Another factor that can explain the discrepancy is the different levels of complexity of the cue-outcome associations between Experiments 2 and 3. In Experiment 2, the cueoutcome association was one color with three quadrants, and in Experiment 3, the cue-outcome association was one color (or texture) with two quadrants. It may be that the cueoutcome association was more complex in Experiment 2 because it involved more quadrants than in Experiment 3, leading to weaker learning of the cue-outcome associations. Indeed, past developmental studies have found that associative learning in infants can be modulated by the complexity of these associations (e.g., Bulf, Johnson, \& Valenza, 2011; Vlach \& Johnson, 2013).

One limitation of the current study is that the current test of the awareness of cue-outcome associations only consisted of four multiple-choice questions. While the group-level accuracy in this test was above chance in the implicit condition, the test accuracy could not adequately measure individual differences in awareness. Future studies can use measures that are more sensitive to individual variability, such as eye movements, to examine the relationship between awareness of cuelocation associations and search performance.

To conclude, we found that people made a conjunctive prediction of the outcome that represented the overlap of the different outcomes previously associated with each cue. This finding reveals a new insight into how the mind makes predictions in novel situations using existing knowledge.

Acknowledgements We would like to thank Chaz Firestone, Justin Halberda, Chris Mole, Yu Luo, Brandon Tomm, and five anonymous reviewers from the Cognitive Science Society for their helpful comments. This work was supported by NSERC Discovery Grant (RGPIN-201405617 to J.Z.), Canada Research Chairs program (to J.Z.), Leaders Opportunity Fund from the Canadian Foundation for Innovation (F1405370 to J.Z.), and Alexander Graham Bell Canada Graduate Scholarships-Doctoral Program (to R.Y.).

Open practices statement The data sets during the current study are available from the authors on reasonable request, and none of the experiments was preregistered.

\section{Compliance with ethical standards}

Conflict of interest None 


\section{Appendix 1 Search accuracy during the prediction phase for all conditions across experiments}

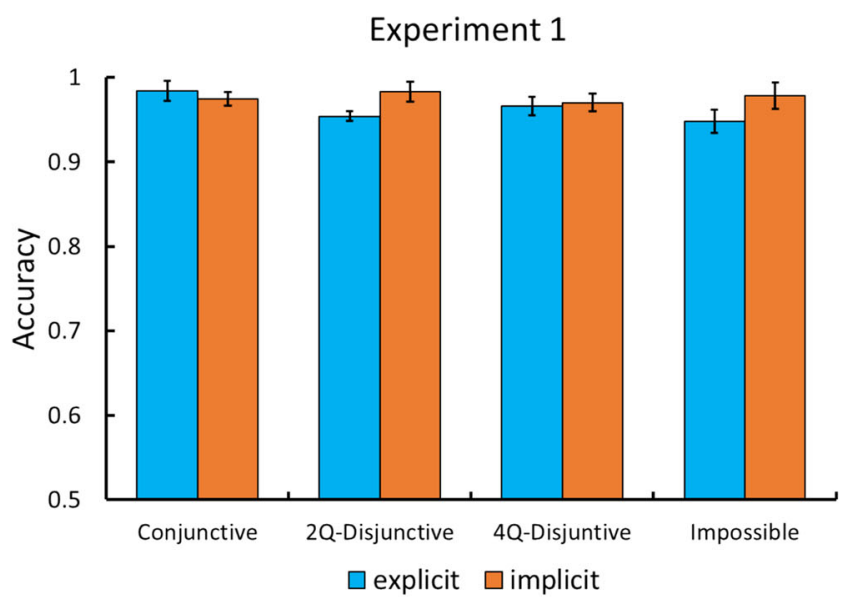

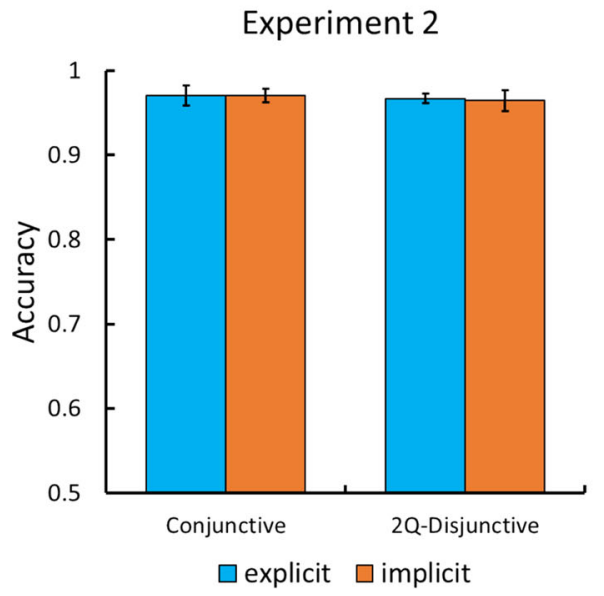

Fig. 5 Accuracy during the prediction phase. The accuracy in different types of locations was separately graphed for Experiments 1, 2, and 3. In each graph, the $x$-axis indicates the type of target location during the prediction phase. The blue bars represent the accuracy in the explicit condition, and the orange bars represent the accuracy in the implicit
Experiment 3

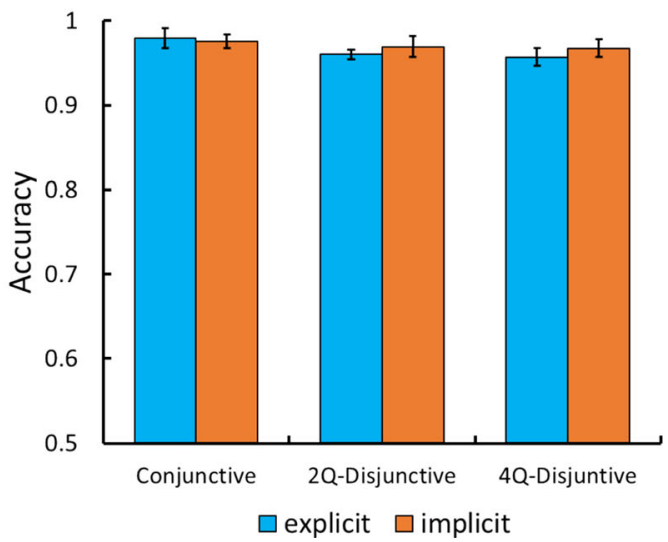

condition. The average accuracy in the search task across all experiments was high (97\%), and there was no reliable difference in accuracy between the conjunction, disjunction, and impossible locations in Experiments 1, 2, and 3. This suggests that there was no speedaccuracy trade-off. Error bars reflect $\pm 1 S E M$. (Color figure online) 


\section{Appendix 2 Search RT over eight epochs in the exposure phase for implicit and explicit conditions in all experiments}
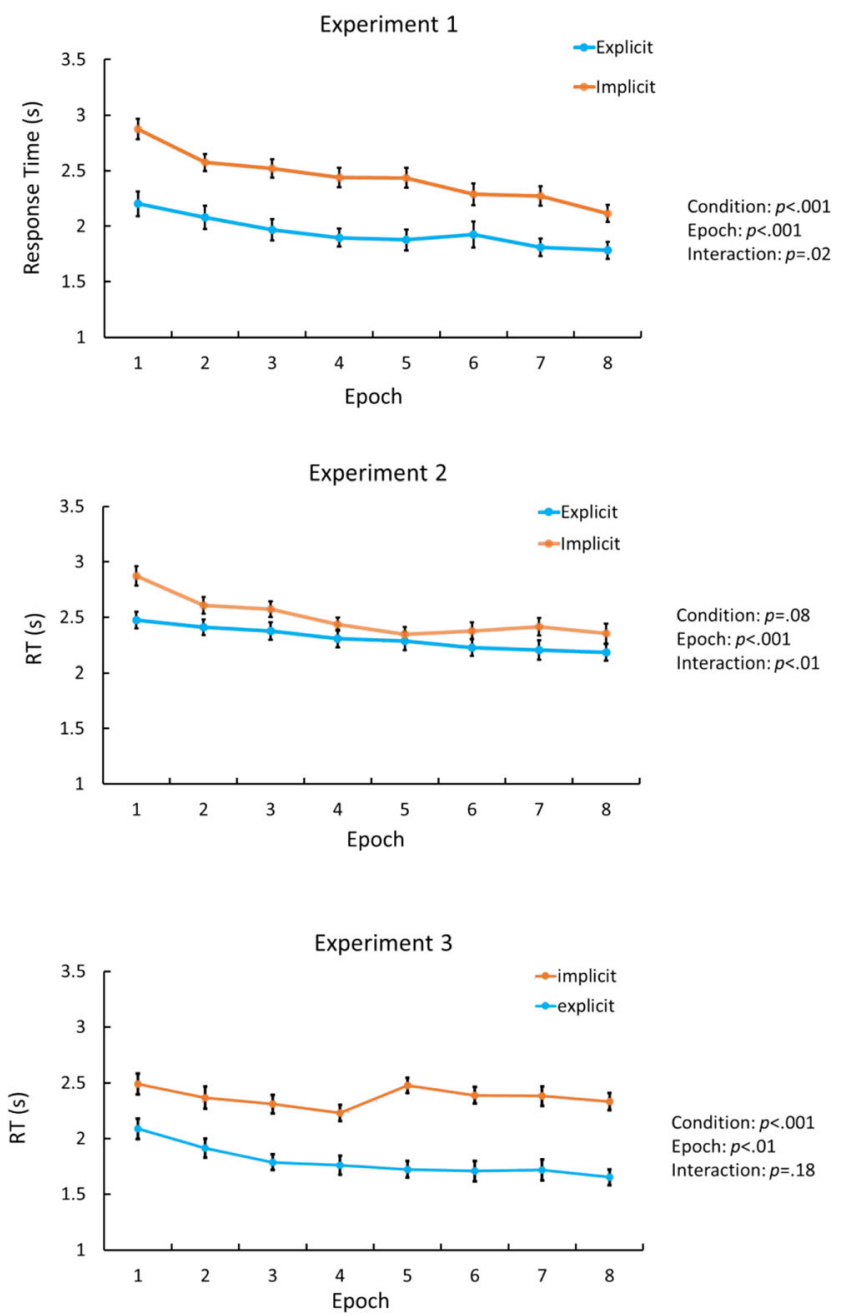

Fig. 6 Time course of RT during the exposure phase. The 160 trials during exposure were divided into eight consecutive epochs (20 trials per epoch), and the RT over the eight epochs was separately graphed for Experiments 1, 2, and 3. The orange lines represent RT in the implicit condition, and the blue lines represent RT in the explicit condition. For all experiments, a 2 (conditions: explicit and implicit, between subjects) $\times 8$ (the eight epochs, within subjects) mixed ANOVA was performed on the search RT data during exposure. In Experiments 1 and 2, search RT in the implicit condition improved more than in the explicit condition, but it was not the case in Experiment 3. Error bars reflect $\pm 1 S E M$. (Color figure online) 


\section{Appendix 3 RT performance for all unique color combinations during the prediction phase in Experiment 1}
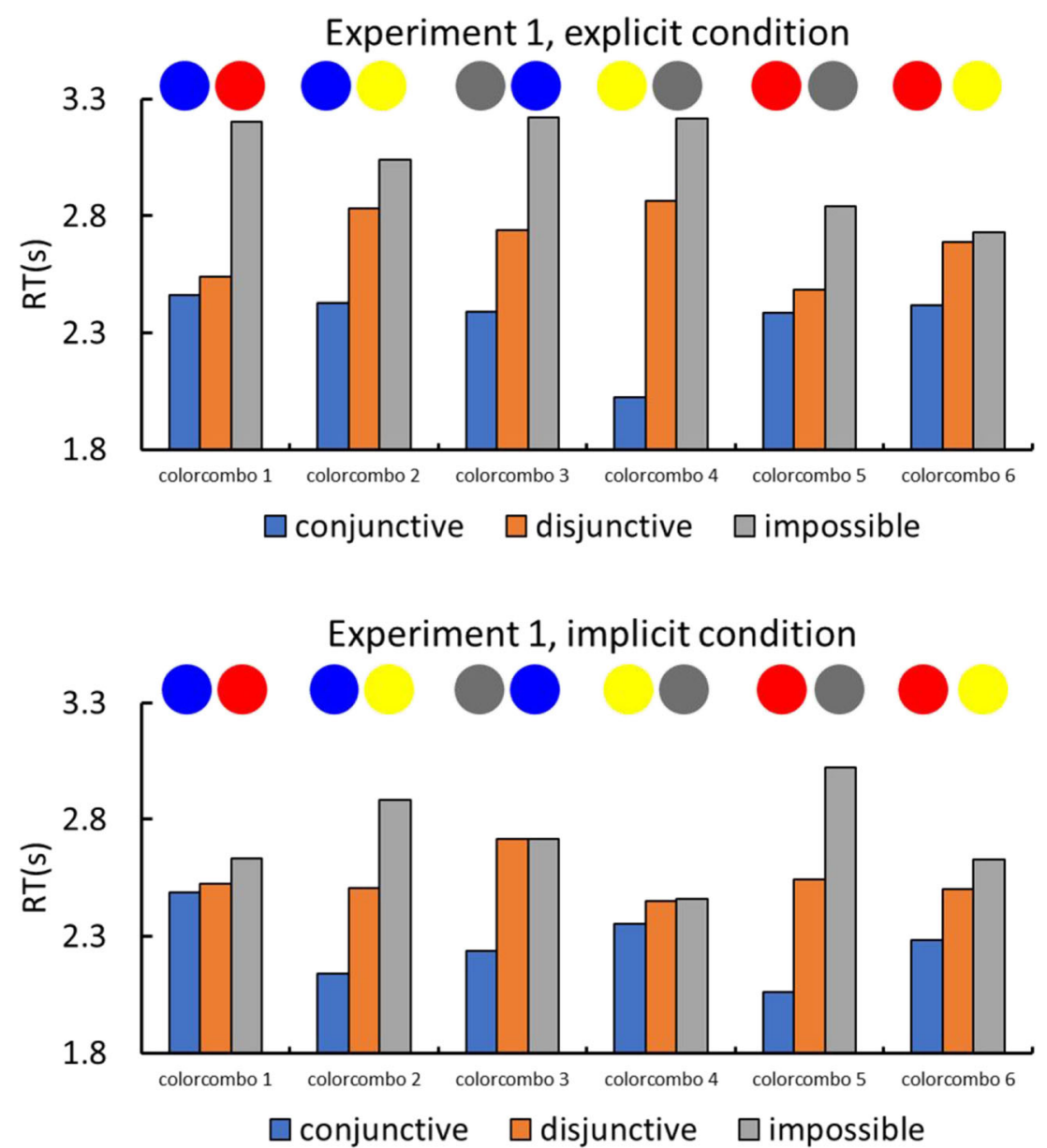

Fig. 7 RT profile for all color combinations. The average RTs for the conjunctive, disjunctive, and impossible locations were graphed for each unique color combination in Experiment 1. The results in explicit and implicit conditions were graphed separately. The dark-blue bars represent the RT in the conjunctive location for each color combination, the darkorange bars represent the RT in the disjunctive location, and the gray bars

\section{References}

Alais, D., \& Burr, D. (2004). The ventriloquist effect results from nearoptimal bimodal integration. Current Biology, 14(3), 257-262. doi: https://doi.org/10.1016/j.cub.2004.01.029

Bulf, H., Johnson, S. P., \& Valenza, E. (2011). Visual statistical learning in the newborn infant. Cognition, 121(1), 127-132. doi:https://oi. org/10.1016/j.cognition.2011.06.010

Chun, M. M., \& Jiang, Y. (1998). Contextual cueing: Implicit learning and memory of visual context guides spatial attention. Cognitive Psychology, 36(1), 28-71. doi:https://doi.org/10.1006/cogp.1998. 0681

Fanselow, M. S., \& Poulos, A. M. (2005). The neuroscience of mammalian associative learning. Annual Review of Psychology, 56, 207234. doi:https://doi.org/10.1146/annurev.psych.56.091103.070213

Faul, F., Erdfelder, E., Lang, A. G., \& Buchner, A. (2007). G*Power 3: A flexible statistical power analysis program for the social, behavioral, represent the RT in the impossible location. Each two-dot color combination is shown above the corresponding RT data. For both implicit and explicit conditions, the RT profiles for the six unique color combinations were consistent, so the effect in the prediction phase was likely not driven by any subset of color combinations. (Color figure online)

and biomedical sciences. Behavior Research Methods, 39, 175-191. doi:https://doi.org/10.3758/BF03193146

Fiser, J., \& Aslin, R. N. (2001). Unsupervised statistical learning of higher-order spatial structures from visual scenes. Psychological Science, 12(6), 499-504. doi:https://doi.org/10.1111/1467-9280. 00392

Hershenson, M. (1962). Reaction time as a measure of intersensory facilitation. Journal of Experimental Psychology, 63(3), 289-293. doi: https://doi.org/10.1037/h0039516

Itti, L., \& Koch, C. (2001). Computational modelling of visual attention. Nature Reviews Neuroscience, 2, 194-203. doi:https://doi.org/10. $1038 / 35058500$

Jiang, Y. V., Swallow, K. M., Rosenbaum, G. M., \& Herzig, C. (2013). Rapid acquisition but slow extinction of an attentional bias in space. Journal of Experimental Psychology: Human Perception and Performance, 39(1), 87-99. doi:https://doi.org/10.1037/a0027611 
Jungé, J. A., Scholl, B. J., \& Chun, M. M. (2007). How is spatial context learning integrated over signal versus noise? A primacy effect in contextual cueing. Visual Cognition, 15(1), 1-11. doi:https://doi. org/10.1080/13506280600859706

Le Pelley, M. E. (2004). The role of associative history in models of associative learning: A selective review and a hybrid model. The Quarterly Journal of Experimental Psychology, 57(3b), 193-243. doi:https://doi.org/10.1080/02724990344000141

Mackintosh, N. J. (1974). The psychology of animal learning. Cambridge, MA: Academic Press.

McGurk, H., \& MacDonald, J. (1976). Hearing lips and seeing voices. Nature, 264, 746-748. doi:https://doi.org/10.1038/264746a0

Mellers, B., Hertwig, R., \& Kahneman, D. (2001). Do frequency representations eliminate conjunction effects? An exercise in adversarial collaboration. Psychological Science, 12(4), 269-275. doi:https:// doi.org/10.1111/1467-9280.00350

Nickerson, R. S. (1973). Intersensory facilitation of reaction time: Energy summation or preparation enhancement? Psychological Review, 80(6), 489-509. doi:https://doi.org/10.1037/h0035437

Raab, D. H. (1962). Statistical facilitation of simple reaction times. Transactions of the New York Academy of Sciences, 24(5), 574 590. doi:https://doi.org/10.1111/j.2164-0947.1962.tb01433.x

Rescorla, R. A., \& Wagner, A. R. (1972). A theory of Pavlovian conditioning: Variations in the effectiveness of reinforcement and nonreinforcement. In A. H. Black \& W. F. Prokasy (Eds.),
Classical conditioning II: Current theory and research (pp. 6499). New York, NY: Appleton-Century-Crofts.

Saffran, J. R., Aslin, R. N., \& Newport, E. L. (1996). Statistical learning by 8-month-old infants. Science, 274(5294), 1926-1928. doi:https:// doi.org/10.1126/science.274.5294.1926

Singer, W., \& Gray, C. M. (1995). Visual feature integration and the temporal correlation hypothesis. Annual Review of Neuroscience, 18, 555-586. doi:https://doi.org/10.1146/annurev.ne.18.030195. 003011.

Treisman, A. M., \& Gelade, G. (1980). A feature-integration theory of attention. Cognitive Psychology, 12(1), 97-136. doi:https://doi.org/ 10.1016/0010-0285(80)90005-5

Tversky, A., \& Kahneman, D. (1983). Extensional versus intuitive reasoning: The conjunction fallacy in probability judgment. Psychological Review, 90(4), 293-315. doi:https://doi.org/10. 1037/0033-295X.90.4.293

Vlach, H. A., \& Johnson, S. P. (2013). Memory constraints on infants' cross-situational statistical learning. Cognition, 127(3), 375-382. doi:https://doi.org/10.1016/j.cognition.2013.02.015

Zhao, J., \& Luo, Y. (2017). Statistical regularities guide the spatial scale of attention. Attention, Perception, \& Psychophysics, 79(1), 24-30. doi:https://doi.org/10.3758/s13414-016-1233-1

Publisher's note Springer Nature remains neutral with regard to jurisdictional claims in published maps and institutional affiliations. 